\title{
Kinematics and excitation mechanisms of molecular hydrogen and [FeII] lines in active galactic nuclei
}

\author{
M. G. Pastoriza ${ }^{1}$, Alberto Rodriguez-Ardila ${ }^{2}$, \\ Sueli Viegas ${ }^{3}$, T. A. A. Sigut ${ }^{4}$ and Anil K. Pradhan ${ }^{5}$ \\ ${ }^{1}$ Departamento de Astronomia, Instituto de Fisica, UFRGS, Av. Bento Gonçalves 9500, C.P. \\ 15051, 91501-970, Porto Alegre, RS, Brazil \\ ${ }^{2}$ Laboratorio Nacional de Astrofisica/CNPq, Brasopolis, MG, Brazil \\ ${ }^{3}$ Instituto de Astronomia, Geofisica e Ciencias Atmosfericas, USP, Sao Paulo, SP, Brazil \\ ${ }^{4}$ Department of Physics and Astronomy, The University of Western Ontario, London, ON, \\ N6A 3K7, Canada \\ ${ }^{5}$ Department of Astronomy, The Ohio State University, Columbus, OH, US 43210-1106, USA
}

\begin{abstract}
Near-infrared spectroscopic observations in the range 0.8-2.4 $\mu \mathrm{m}$ are used to study the kinematics and excitation mechanisms of Molecular Hydrogen and [FeII] lines in a sample of AGNs. The width of $\mathrm{H}_{2}$ lines is narrower than [FeII] lines, therefore they do not originate from the same parcel of gas. The molecular emission is found to be purely thermal but with heating processes which vary among the objects. A clear correlation between $\mathrm{H}_{2} / \mathrm{Br} \gamma$ and [FeII] $1.257 \mu \mathrm{m} / \mathrm{Pa} \beta$ is found for our sample objects supplemented with data from the literature. The correlation of these line ratios is a useful diagnostic tool in the NIR to separate emitting line objects by their level of nuclear activity.
\end{abstract}

\section{Introduction}

One of the fundamental problems in active galactic nuclei (AGN) research is to determine the dominant excitation mechanisms of the narrow line emitting gas, i.e. whether it is due to non-stellar processes (e.g. photoionization from a central source or shocks from a radio jet) or to stellar processes (e.g. photoionization from OB stars or shocks from supernova remnants). This ambiguity is most evident when interpreting the spectral lines of low-ionization species such as [Fe II] and molecular hydrogen. Both sets of lines are detected in galaxies displaying varying degrees of nuclear activity, from objects classified as starburst-dominated (SD) to those classified as AGN-dominated. Are these lines produced by a unique mechanism in the objects where they are observed, evidencing the so-called AGN-starburst connection? or are they formed by different mechanisms (or some combination thereof) in different classes of objects?

\section{Observations}

Near-infrared (NIR) spectra from 0.8-2.4 $\mu \mathrm{m}$ were obtained at the NASA $3 \mathrm{~m}$ Infrared Telescope Facility (IRTF) with the SpeX spectrometer (Rayner et al. 2003). A 0.8 " $\times 15$ " slit was used during the observations, giving a spectral resolution of $360 \mathrm{~km} \mathrm{~s}^{-1}$. For the objects in which the host galaxy is clearly detected on the DSS images, the slit was oriented along the major axis of the target. Table 1 lists the galaxies in order of right ascension. 
Table 1. Observations log and basic properties of the sample. The objects are listed in order of right ascension.

\begin{tabular}{|c|c|c|c|c|c|c|c|c|c|}
\hline $\begin{array}{l}\text { ID } \\
\text { (1) }\end{array}$ & $\begin{array}{l}\text { Galaxy } \\
(2)\end{array}$ & $\begin{array}{c}\text { Type } \\
(3)\end{array}$ & $\begin{array}{c}z \\
(4)\end{array}$ & $\begin{array}{c}\mathrm{E}(\mathrm{B}-\mathrm{V}) \mathrm{G} \\
(5)\end{array}$ & $\begin{array}{c}\text { Date of } \\
\text { Observation } \\
(6)\end{array}$ & $\begin{array}{c}\text { On-source } \\
\text { Integration } \\
\text { tine }(\mathrm{s}) \\
(7)\end{array}$ & $\begin{array}{l}\text { Airmass } \\
(8)\end{array}$ & $\begin{array}{l}\text { PA } \\
(\text { deg }) \\
(9)\end{array}$ & $\begin{array}{l}\mathrm{r}^{\mathrm{a}} \\
\text { (pc) } \\
(10)\end{array}$ \\
\hline 1 & Mrk 1210 & Sy 2 & 0.01406 & 0.030 & 2002 Apr 25 & 2700 & 1.25 & 58 & 220 \\
\hline 2 & Mrk 124 & NLS1 & 0.05710 & 0.015 & 2002 Apr 23 & 2640 & 1.16 & 10 & 990 \\
\hline 3 & Mrk 1239 & NLS1 & 0.01927 & 0.065 & $\begin{array}{l}2002 \text { Apr } 21 \\
2002 \text { Apr } 23\end{array}$ & $\begin{array}{l}1920 \\
1920\end{array}$ & $\begin{array}{l}1.08 \\
1.15\end{array}$ & $\begin{array}{l}0.0 \\
0.0\end{array}$ & 335 \\
\hline 4 & NGC 3227 & Sy 1 & 0.00386 & 0.023 & $\begin{array}{l}2002 \text { Apr } 21 \\
2002 \text { Apr } 25\end{array}$ & $\begin{array}{c}720 \\
1080\end{array}$ & $\begin{array}{l}1.00 \\
1.02\end{array}$ & $\begin{array}{l}158 \\
158\end{array}$ & 67 \\
\hline 5 & H1143-192 & Sy 1 & 0.03330 & 0.039 & 2002 Apr 21 & 1920 & 1.31 & 45 & 520 \\
\hline 6 & NGC 3310 & SB & 0.00357 & 0.022 & 2002 Apr 21 & 840 & 1.21 & 158 & 56 \\
\hline 7 & NGC 4051 & NLS1 & 0.00234 & 0.013 & 2002 Apr 20 & 1560 & 1.17 & 132 & 37 \\
\hline 8 & NGC 4151 & Sy 1 & 0.00345 & 0.028 & 2002 Apr 23 & 1800 & 1.10 & 130 & 58 \\
\hline 9 & Mrk 766 & NLS1 & 0.01330 & 0.020 & $\begin{array}{l}2002 \text { Apr } 21 \\
2002 \text { Apr } 25\end{array}$ & $\begin{array}{l}1680 \\
1080\end{array}$ & $\begin{array}{l}1.06 \\
1.02\end{array}$ & $\begin{array}{l}112 \\
112\end{array}$ & 230 \\
\hline 10 & NGC 4748 & NLS1 & 0.01417 & 0.052 & $\begin{array}{l}2002 \text { Apr } 21 \\
2002 \text { Apr } 25\end{array}$ & $\begin{array}{l}1680 \\
1440\end{array}$ & $\begin{array}{l}1.29 \\
1.21\end{array}$ & $\begin{array}{l}36 \\
36\end{array}$ & 254 \\
\hline 11 & Mrk 279 & NLS1 & 0.03068 & 0.016 & 2002 Apr 24 & 3600 & 1.54 & 0.0 & 480 \\
\hline 12 & NGC 5548 & Sy1 & 0.01717 & 0.020 & 2002 Apr 23 & 1920 & 1.05 & 112 & 298 \\
\hline 13 & Mrk 478 & NLS1 & 0.07760 & 0.014 & 2002 Apr 20 & 3240 & 1.06 & 0.0 & 1200 \\
\hline 14 & NGC 5728 & Sy2 & 0.01003 & 0.101 & 2002 Apr 21 & 960 & 1.31 & 36 & 160 \\
\hline 15 & PG $1448+273$ & QSO & 0.06522 & 0.029 & 2002 Apr 24 & 2160 & 1.01 & 108 & 1020 \\
\hline 16 & Mrk 291 & NLS1 & 0.03519 & 0.038 & 2002 Apr 21 & 2520 & 1.04 & 84 & 550 \\
\hline 17 & Mrk 493 & NLS1 & 0.03183 & 0.025 & $\begin{array}{l}2002 \text { Apr } 20 \\
2002 \text { Apr } 25\end{array}$ & $\begin{array}{c}1800 \\
900\end{array}$ & $\begin{array}{l}1.07 \\
1.04\end{array}$ & $\begin{array}{l}0.0 \\
0.0\end{array}$ & 500 \\
\hline 18 & PG $1612+261$ & QSO & 0.13096 & 0.054 & 2002 Apr 23 & 2520 & 1.10 & 107 & 2050 \\
\hline 19 & Mrk 504 & NLS1 & 0.03629 & 0.050 & 2002 Apr 21 & 2100 & 1.04 & 138 & 570 \\
\hline 20 & $1 \mathrm{H} 1934-063$ & NLS1 & 0.01059 & 0.293 & $\begin{array}{l}2002 \text { Apr } 21 \\
2002 \text { Apr } 25\end{array}$ & $\begin{array}{c}1200 \\
720\end{array}$ & $\begin{array}{l}1.13 \\
1.17\end{array}$ & $\begin{array}{l}150 \\
150\end{array}$ & 170 \\
\hline 21 & Mrk 896 & NLS1 & 0.02678 & 0.045 & $\begin{array}{l}2002 \text { Apr } 23 \\
2002 \text { Apr } 24 \\
2002 \text { Apr } 25\end{array}$ & $\begin{array}{l}1440 \\
1200 \\
1200\end{array}$ & $\begin{array}{l}1.21 \\
1.18 \\
1.17\end{array}$ & $\begin{array}{l}150 \\
150 \\
150\end{array}$ & 420 \\
\hline 22 & Ark 564 & NLS1 & 0.02468 & 0.060 & 2000 Oct 10 & 1500 & 1.05 & 0.0 & 390 \\
\hline
\end{tabular}

a Radius of the integrated region.

\section{Kinematics of the $H_{2}$ and [FeII]}

The large spectral coverage and medium spectral resolution of our data $\left(360 \mathrm{~km} \mathrm{~s}^{-1}\right)$ allow us to discuss how the widths of the $\mathrm{H}_{2}$ lines compares to that of other narrow lines. In Figure 1 we presents a camparison of the lines profile of [FeII] $1.257 \mu \mathrm{m}$ (full histogram) and $\mathrm{H}_{2} 2.122 \mu \mathrm{m}$ for Mrk 1210, for which the observed profile of $\mathrm{Br} \gamma$ (solid line) has also been plotted. The smaller values of the FWHM of $\mathrm{H}_{2}$ relative to other narrow lines suggest that the molecular gas is concentrated in the external/extended NLR or possibly even in the host galaxy, where the gravitational effects of the black hole on the emitting gas are lower than in the BLR. This scenario seems unlikely, however, because for the large majority of the objects the size of the integrated region covered by the spectra is no larger than $500 \mathrm{pc}$. The alternative is to consider that the molecular gas is not kinematically linked to the NLR gas even though the two are co-spatial.

\section{Excitation mechanisms of the NIR $H_{2}$ lines}

The Fig. 2 shows:(a) 2-1S(1)/1-0S(1) vs 1-0S(2)/1-0S(0) and (b) 2-1S(1)/1-0S(1) vs 1-0S(3)/1-0S(1). These diagnostic diagrams have been proposed by Mouri (1994). Curves represent thermal emission at 500-3000 K. Crosses are the non-thermal UV excitation models of Black \& Van Dishoek (1987). Open circles are thermal UV excitation models of Sternberg \& Dalgarno (1989). The numbers identify each object according to the notation given in column 1 of Table 1 . The black square in (a) represents the datum for NGC 253, a typical starburst galaxy, taken from Harrison et al. (1998). A large scatter is observed in both line ratios for the galaxy sample. NGC 3227, NGC 4051, NGC 4151 and Mrk 766 (labelled 4, 7, 8 and 9, respectively) fall near the thermal curve model and indicate excitation temperatures for the thermal component of between 1500 and $2500 \mathrm{~K}$. For these galaxies, the $\mathrm{H}_{2}$ emission can be considered purely thermal. For the remaining 


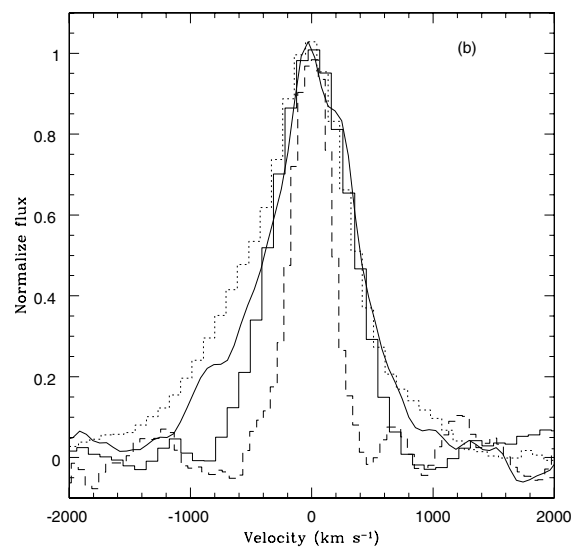

Figure 1. Comparation of the line profiles of [FeII] $1.257 \mu \mathrm{m}$ (full histogram) and $H_{2}$ $2.122 \mu \mathrm{m}$ for Mrk 1210, for which the oberved profile of $\operatorname{Br} \gamma$ (solid line) has also been plotted.
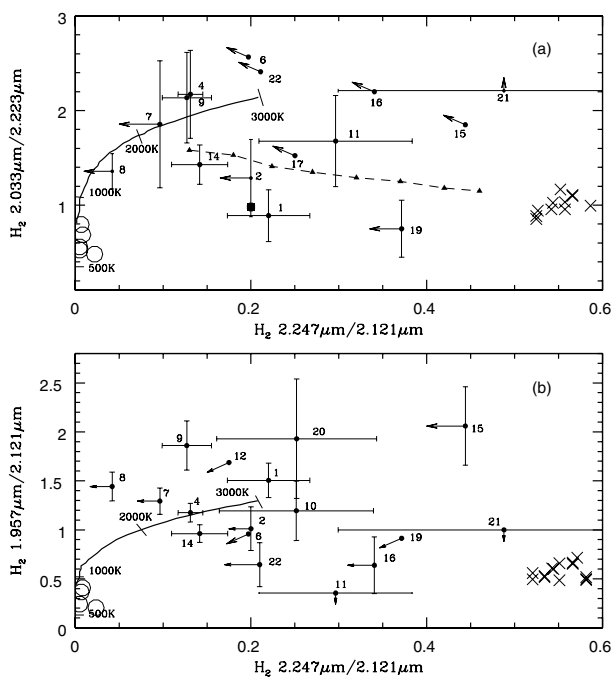

Figure 2. Plot of (a) $2-1 \mathrm{~S}(1) / 1-0 \mathrm{~S}(1)$ vs $1-0 \mathrm{~S}(2) / 1-0 \mathrm{~S}(0)$ and (b) $2-1 \mathrm{~S}(1) / 1-0 \mathrm{~S}(1)$ vs 1-0S(3)/1-0S(1). Curves represents thermal emission at 500-3000 K. Crosses are the non-thermal UV excitation models of Black \& Van Dishoek (1987). Open circles are thermal UV excitation models of Sternberg \& Dalgarno (1989). The numbers identify each object according to the notation given in column 1 of Table 1 . The black square in (a) represents the datum for NGC 253, a typical starburst galaxy, taken from Harrison et al.(1998)

objects, a mixture of thermal and non-thermal processes are probably at work, with the excitation temperature of the thermal component higher than $1000 \mathrm{~K}$. Interestingly, no AGN is located in the region occupied by the pure non-thermal UV excitation models, except possibly Mrk 896 


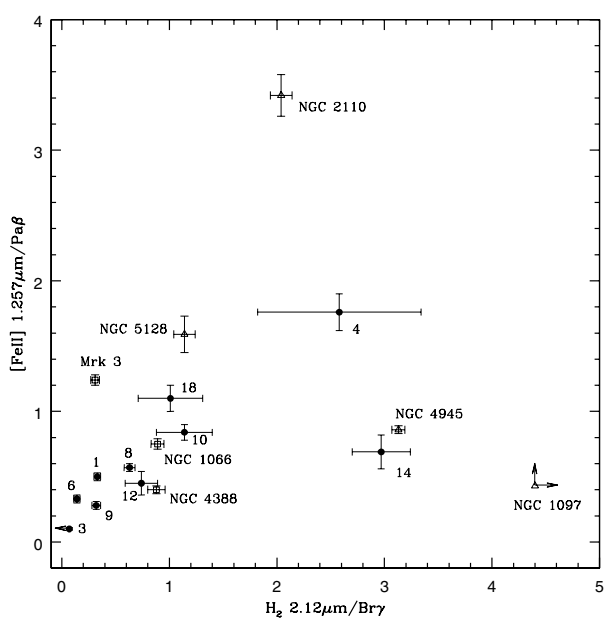

Figure 3. The ratios $\mathrm{H}_{2} 2.121 \mu \mathrm{m} / \mathrm{Br} \gamma$ and [FeII] $1.257 \mu \mathrm{m} / \mathrm{Pa} \beta$. Only the Seyfert $1 \mathrm{~s}$ and NLS1 in which a reliable deblending of the narrow component of the H I lines was carried out are plotted. Filled circles represents our data and open symbols data taken from the literature. Triangles are measurements from Knop et al. (1996) and squares from Reunanen et al (2002). The number along each data point identifies our galaxies according to the notation given in column 1 of Table 1.

\section{Are the $[\mathrm{FeII}]$ and $H_{2}$ related}

The observational evidence indicates that [FeII] and $\mathrm{H}_{2}$ are common features in the nuclear spectra of AGN, although probably originated from different parcels of gas. However, this does not exclude the possibility that both sets of lines originate from a single, dominant mechanism. Moreover, if dust is intermixed with the line emitting clouds, and there are strong velocity gradients, $[\mathrm{FeII}]$ and $\mathrm{H}_{2}$ lines can appear to have different velocity fields even though they are produced in adjacent regions.

If [FeII] and $\mathrm{H}_{2}$ have a common origin, a strong correlation between their observed fluxes should be expected. The Figure shows the correlation between $\mathrm{H}_{2} 2.121 \mu \mathrm{m} / \mathrm{Br} \gamma$ and [FeII] $1.257 \mu \mathrm{m} / \mathrm{Pa} \beta$ for our sample. Only the Seyfert $1 \mathrm{~s}$ and NLS1 in which a reliable deblending of the narrow component of the HI lines was carried out are plotted in Fig. 3. Filled circles represent our data and open symbols data taken from the literature. Triangles are measurements from Knop et al (1996). and squares from Reunanen et al. (2002). The number along each data point identifies our galaxies according to the notation given in column 1 of Table 1.

\section{References}

Black, J. H., \& van Dishoeck, E. F. 1987, ApJ, 322, 412

Harrison, A., Puxley, P., Russel, A., \& Brand, P. 1998, MNRAS, 297, 624

Knop, R. A., Armus, L., Larkin, J. E., Matthews, K., Shupe, D. L., \& Soifer, B. T. 1996, AJ, 112,81

Mouri, H. 1994, ApJ, 427, 777

Rayner, J. T., et al. 2003, PASP, 155, 362

Reunanen, J., Kotilainen, J. K., \& Prieto, M. A. 2002, MNRAS, 331, 154

Sternberg, A., \& Dalgarno, A. 1989, ApJ, 338, 197 